\title{
32. Two-dimensional generalized thermo-elastic problem for anisotropic half-space
}

\author{
Debkumar Ghosh ${ }^{1}$, Abhijit Lahiri' ${ }^{2}$ Ibrahim A. Abbas ${ }^{3}$ \\ ${ }^{1,2}$ Department of Mathematics, Jadavpur University, Kolkata, 700032, India \\ ${ }^{3}$ Department of Mathematics, Sohag University, Sohag, Egypt \\ ${ }^{1}$ Corresponding author \\ E-mail: ${ }^{1}$ debkumarghosh2020@gmail.com, ${ }^{2}$ lahiriabhijit2000@yahoo.com, ${ }^{3}$ ibrabbas7@yahoo.com
}

Received 8 February 2017; accepted 12 March 2017

DOI https://doi.org/10.21595/mme.2017.18236

Check for updates

\begin{abstract}
This paper concerns with the study of wave propagation in fibre reinforced anisotropic half space under the influence of temperature and hydrostatic initial stress. Lord-Shulman theory is applied to the heat conduction equation. The resulting equations are written in the form of vector matrix differential equation by using Normal Mode technique, finally which is solved by Eigen value approach.
\end{abstract}

Keywords: eigenvalue, generalized thermoelasticity, normal mode, vector-matrix differential equation.

\section{Nomenclature}

$\begin{array}{ll}u_{i} & \text { Displacement tensor } \\ t & \text { Time variable } \\ \sigma_{i j} & \text { Stress components } \\ \rho & \text { Mass density } \\ e_{i j} & \text { Strain components } \\ \omega_{i j} & \text { Rotational tensor } \\ T & \text { Temperature } \\ T_{0} & \text { Reference temperature } \\ \beta_{i j} & \text { Thermal elastic coupling tensor } \\ c_{e} & \text { Specific heat at constant strain } \\ K_{i j} & \text { Thermal conductivity } \\ P & \text { Initial pressure } \\ t_{0} & \text { Relaxation time } \\ \delta_{i j} & \text { Kronecker Delta } \\ \lambda, \mu_{T} & \text { Elastic parameters } \\ \alpha, \beta,\left(\mu_{L}-\mu_{T}\right) & \text { Reinforced elastic parameter }\end{array}$

\section{Introduction}

Fibre-reinforced composite(FRC) materials are usually low weight and high strength used in construction engineering. The physical property of FRC material is governed by the theory of elasticity for different materials with the direction along the direction of fibre. Green [1] studied wave propagation in anisotropic elastic plates. Abbas and Othman [2] discussed the distribution of wave propagation under hydrostatic initial stress of fibre-reinforced materials in anisotropic half-space. Baylies and Green [3] analyse the flexural waves in fibre-reinforced laminated plates. Rogerson [4] discussed effect of penetration in a six-ply composite laminates.

Most of the thermoelasticity and generalized thermoelasticity (coupled or uncoupled) problems have been solved by potential function approach. This method is not always suitable as discussed by Dhaliwal and Sherief [5] and Sherief and Anwar [6]. These may be summarized by the initial conditions and the boundary conditions for physical problems which are directly concern with the material quantities under consideration and not with the potential function. Also, the potential function representations are not convergent always while the physical problems in 
natural variables constitute convergent solution. So, the alternative method of potential function approach is eigenvalue approach. In this method, we obtain a vector-matrix differential equation from the basic equations which reduces finally to an algebraic eigenvalue problem and the solutions for the field variables are obtained by determining the eigenvalues and eigenvectors from the corresponding coefficient matrix. In this theory, body forces and/or heat sources are also accommodated as in Das and Lahiri [7], Bachher et al. [8]. Now, two different models of generalized thermoelastisities are extensively used. One is Lord and Shulman (L-S) [9] theory and the other is Green and Lindsay (G-L) [10] theory. Introducing one relaxtion time parameter in L-S theory the heat conduction equation becomes hyperbolic type without violating conventional Fourier's law. Whereas the G-L theory modified the heat conduction equation as well as the equation of motion in coupled thermoelasticitywo relaxation time parameters. There are other three models (Model I, II and III by Green and Nagdhi [11-13]) for generalized thermoelasticity concerned to the theory of with or without energy dissipation.

\section{Development of governing equations}

The stress-strain relation and the governing equations of motion without body forces and heat sources are written as follow:

$$
\begin{aligned}
& \sigma_{i j, j}-P \omega_{i j, j}=\rho \ddot{u}_{i}, \\
& \sigma_{i j}=\lambda e_{k k} \delta_{i j}+2 \mu_{T} e_{i j}+\alpha\left(a_{k} a_{m} e_{k m} \delta_{i j}+a_{i} a_{j} e_{k k}\right) \\
& \quad+2\left(\mu_{L}-\mu_{T}\right)\left(a_{i} a_{k} e_{k j}+a_{j} a_{k} e_{k i}\right)+\beta a_{k} a_{m} e_{k m} a_{i} a_{j}-\beta_{i j}\left(T-T_{0}\right) \delta_{i j}, \quad i, j, k, m=1,2,3, \\
& e_{i j}=\frac{1}{2}\left(u_{i, j}+u_{j, i}\right) \\
& \omega_{i j}=\frac{1}{2}\left(u_{j, i}-u_{i, j}\right) \\
& K_{i j} T_{i j}=\rho c_{e}\left(\dot{T}+t_{0} \ddot{T}\right)+T_{0}\left(\dot{u}_{i, j} \ddot{u}_{i, j}\right), \quad i, j=1,2,3 .
\end{aligned}
$$

We consider the problem of a elastic half-space $(x \geq 0)$ in fibre-reinforced anisotropic material with $a \equiv\left(a_{1}, a_{2}, a_{3}\right)$ where $a_{1}^{2}+a_{2}^{2}+a_{3}^{2}=1$ as in I. A. Abbas [14], where the displacements are given:

$$
u=u_{x}=u(x, y, t), v=u_{y}=v(x, y, t), \quad w=u_{z}=0 \text {. }
$$

We consider the direction of fibre as $a \equiv(1,0,0)$ with $x$-axis as prefered direction, and Eqs. (1-5), reduces as given:

$$
\begin{aligned}
& \sigma_{11}=\left(\lambda+2 \alpha+4 \mu_{L}-2 \mu_{T}+\beta\right) \frac{\partial u}{\partial x}+(\lambda+\alpha) \frac{\partial v}{\partial y}-\beta_{11}\left(T-T_{0}\right), \\
& \sigma_{22}=\left(\lambda+2 \mu_{T}\right) \frac{\partial v}{\partial y}+(\alpha+\lambda) \frac{\partial u}{\partial x}-\beta_{22}\left(T-T_{0}\right), \\
& \sigma_{12}=\mu_{L}\left(\frac{\partial v}{\partial x}+\frac{\partial u}{\partial y}\right), \\
& A_{11} \frac{\partial^{2} u}{\partial x^{2}}+\left(A_{12}+\mu_{L}-\frac{P}{2}\right) \frac{\partial^{2} v}{\partial x \partial y}+\left(\mu_{L}+\frac{P}{2}\right) \frac{\partial^{2} u}{\partial y^{2}}-\beta_{11} \frac{\partial T}{\partial x}=\rho \frac{\partial^{2} u}{\partial t^{2}}, \\
& A_{22} \frac{\partial^{2} v}{\partial y^{2}}+\left(A_{12}+\mu_{L}-\frac{P}{2}\right) \frac{\partial^{2} u}{\partial x \partial y}+\left(\mu_{L}+\frac{P}{2}\right) \frac{\partial^{2} v}{\partial x^{2}}-\beta_{22} \frac{\partial T}{\partial y}=\rho \frac{\partial^{2} v}{\partial t^{2}}, \\
& K_{11} \frac{\partial^{2} T}{\partial x^{2}}+K_{22} \frac{\partial^{2} T}{\partial y^{2}}=\left(\frac{\partial}{\partial t}+t_{0} \frac{\partial^{2}}{\partial t^{2}}\right)\left(\rho c_{e} T+T_{0} \beta_{11} \frac{\partial u}{\partial x}+T_{0} \beta_{22} \frac{\partial v}{\partial y}\right),
\end{aligned}
$$


with:

$A_{11}=\lambda+2\left(\alpha+\mu_{L}\right)+4\left(\mu_{L}-\mu_{T}\right)+\beta, \quad A_{12}=\alpha+\lambda, \quad A_{22}=\lambda+2 \mu_{T}$,

$\beta_{11}=\left(2 \lambda+3 \alpha+4 \mu_{L}-2 \mu_{T}+\beta\right) \alpha_{11}+(\lambda+\alpha) \alpha_{22}, \quad \beta_{22}=(2 \lambda+\alpha) \alpha_{11}+\left(\lambda+2 \mu_{T}\right) \alpha_{22}$,

where $\alpha_{11}, \alpha_{22}$ are linear thermal expansion coeeficients.

To transform the above governing equations in non-dimensional forms, we introduce the nondimensional variables as follows:

$$
\begin{aligned}
& \left(x^{\prime}, y^{\prime}, u^{\prime}, v^{\prime}\right)=c_{1} \chi(x, y, u, v), t^{\prime}=c_{1}^{2} \chi t, \quad T^{\prime}=\frac{\beta_{11}\left(T-T_{0}\right)}{\rho c_{1^{2}}}, \quad \chi=\frac{\rho c_{e}}{K_{11}}, \\
& \left(\sigma^{\prime}{ }_{11}, \sigma^{\prime}{ }_{12}, \sigma^{\prime}{ }_{22}\right)=\frac{1}{\rho c_{1}^{2}}\left(\sigma_{11}, \sigma_{12}, \sigma_{22}\right), \quad c_{1}^{2}=\frac{A_{11}}{\rho} .
\end{aligned}
$$

Using non-dimensional Eq. (13), the governing equations reduces to (eleminating primes for convenience):

$$
\begin{aligned}
& \sigma_{11}=\frac{\partial u}{\partial x}+B_{1} \frac{\partial v}{\partial y}-T, \\
& \sigma_{22}=B_{1} \frac{\partial u}{\partial x}+B_{2} \frac{\partial v}{\partial y}-B_{3} T \\
& \sigma_{12}=B_{4}\left(\frac{\partial v}{\partial x}+\frac{\partial u}{\partial y}\right), \\
& \frac{\partial^{2} u}{\partial x^{2}}+\left(B_{1}+B_{4}-\frac{R_{p}}{2}\right) \frac{\partial^{2} v}{\partial x \partial y}+\left(B_{4}+\frac{R_{p}}{2}\right) \frac{\partial^{2} u}{\partial y^{2}}-\frac{\partial T}{\partial x}=\frac{\partial^{2} u}{\partial t^{2}}, \\
& B_{2} \frac{\partial^{2} v}{\partial y^{2}}+\left(B_{1}+B_{4}-\frac{R_{p}}{2}\right) \frac{\partial^{2} u}{\partial x \partial y}+\left(B_{4}+\frac{R_{p}}{2}\right) \frac{\partial^{2} v}{\partial x^{2}}-B_{3} \frac{\partial T}{\partial y}=\frac{\partial^{2} v}{\partial t^{2}}, \\
& \frac{\partial^{2} T}{\partial x^{2}}+\varepsilon_{1} \frac{\partial^{2} T}{\partial y^{2}}=\left(\frac{\partial}{\partial t}+t_{0} \frac{\partial^{2}}{\partial t^{2}}\right)\left(T+\varepsilon_{2} \frac{\partial u}{\partial x}+\varepsilon_{3} \frac{\partial v}{\partial y}\right),
\end{aligned}
$$

where:

$$
\begin{aligned}
& \left(B_{1}, B_{2}, B_{3}\right)=\frac{1}{A_{11}}\left(A_{12}, A_{22}, \mu_{1}\right), \quad B_{3}=\frac{\beta_{22}}{\beta_{11}}, \quad R_{p}=\frac{P}{A_{11}}, \\
& \left(\varepsilon_{2}, \varepsilon_{3}\right)=\frac{T_{0} \beta_{11}}{A_{11} \rho c_{e}}\left(\beta_{11}, \beta_{22}\right), \quad \varepsilon_{1}=\frac{K_{11}}{K_{22}} .
\end{aligned}
$$

\section{Solution procedure}

\subsection{Normal mode analysis: formulation of vector-matrix differential equation}

For the solution of the Eqs. (14-19), physical variables can be decomposed using normal modes Eq. (20) in the following form:

$\left[u, v, T, \sigma_{11}, \sigma_{12}, \sigma_{22}\right](x, y, t)=\left[u^{*}, v^{*}, T^{*}, \sigma_{11}^{*}, \sigma_{12}^{*}, \sigma_{22}^{*}\right](x) e^{\omega t+i a y}$,

where $i=\sqrt{-1}, \omega$ is the angular frequency and a is the wave number along $x$-axis.

Using Eq. (20), Eqs. (14-19) reduces to omitting '*' for convenience:

$\sigma_{11}=\frac{d u}{d x}+\left(B_{1} i a\right) v-T$ 
$\sigma_{22}=B_{1} \frac{d u}{d x}+\left(B_{2} i a\right) v-B_{3} T$

$\sigma_{12}=B_{4} \frac{d v}{d x}+\left(B_{4} i a\right) u$,

$\frac{d^{2} u}{d x^{2}}=M 41 . u+0 . v+0 . T+0 . u^{\prime}+M 45 \cdot v^{\prime}+T^{\prime}$,

$\frac{d^{2} v}{d x^{2}}=0 . u+M 52 . v+M 53 . T+M 54 . u^{\prime}+0 . v^{\prime}+0 . T^{\prime}$

$\frac{d^{2} T}{d x^{2}}=0 . u+M 62 . v+M 63 . T+M 64 . u^{\prime}+0 . v^{\prime}+0 . T^{\prime}$,

where:

$M 41=a^{2}\left[B_{4}+\frac{R_{p}}{2}\right]+\omega^{2}, \quad M 45=-i a\left[B_{1}+B_{4}-\frac{R_{p}}{2}\right], \quad M 52=\frac{a^{2} B_{2}+\omega^{2}}{B_{4}+\frac{R_{p}}{2}}$,

$M 53=\frac{\frac{R_{p}}{2}-B_{1}-B_{4}}{B_{4}+\frac{R_{p}}{2}}, \quad M 54=\frac{i a B_{3}}{B_{4}+\frac{R_{p}}{2}}, \quad M 62=i a \varepsilon_{3}\left(\omega+t_{0} \omega^{2}\right)$,

$M 63=\omega+t_{0} \omega^{2}+\varepsilon_{2} a^{2}, \quad M 64=\varepsilon_{2}^{2}\left(\omega+t_{0} \omega^{2}\right)$.

Eqs. (24-26) can be written in the form of vector-matrix differential equation as $[2,8]$ :

$\frac{d \vec{W}}{d x}=\vec{A} \vec{W}$

where $\vec{W}=\left[\begin{array}{lllllll}u & v & T & u^{\prime} & v^{\prime} & T^{\prime}\end{array}\right]^{T}$ and $A=\left[\begin{array}{ll}L_{11} & L_{12} \\ L_{21} & L_{22}\end{array}\right]$. Where $L_{11}$ is null matrix and $L_{12}$ identity matrix of order $3 \times 3$ respectively and $L_{21}$ and $L_{22}$ are given by:

$L_{11}=\left(\begin{array}{lll}0 & 0 & 0 \\ 0 & 0 & 0 \\ 0 & 0 & 0\end{array}\right), \quad L_{12}=\left(\begin{array}{lll}1 & 0 & 0 \\ 0 & 1 & 0 \\ 0 & 0 & 1\end{array}\right)$,

$L_{21}=\left(\begin{array}{ccc}M 41 & 0 & 0 \\ 0 & M 52 & M 53 \\ 0 & M 62 & M 63\end{array}\right), \quad L_{22}=\left(\begin{array}{ccc}0 & M 45 & 1 \\ M 54 & 0 & 0 \\ M 64 & 0 & 0\end{array}\right)$.

\subsection{Solution of the vector-matrix differential equation}

To solve the vector-matrix differential Eq. (27), we apply the method of eigenvalue approach,

The characteristic equation of the matrix $\vec{A}$ is given by:

$|A-\lambda I|=0$

The roots of the characteristic Eq. (28) are $\lambda=\lambda_{i}, i=1,2,3$ which are of the form $\lambda= \pm \lambda_{1}$, $\lambda= \pm \lambda_{2}$ and $\lambda= \pm \lambda_{3}$ and they are also eigenvalues of the matrix.

The eigenvector, $\vec{W}$ corresponding to the eigenvalue $\lambda$ can obtained as:

$\vec{X}_{\lambda}=\left[\begin{array}{llllll}\delta_{1} & \delta_{2} & \delta_{3} & \lambda \delta_{1} & \lambda \delta_{2} & \lambda \delta_{3}\end{array}\right]^{T}$, 
where $\delta_{1}=a_{2} b_{3}-a_{3} b_{2}, \delta_{2}=a_{3} b_{1}-a_{1} b_{3}, \delta_{3}=a_{1} b_{2}-a_{2} b_{1}$.

As in Lahiri et al. [7], the general solution of Eq. (27) which is regular as can be written as:

$\vec{W}=\sum_{i=1}^{3} A_{i} X_{i} e^{-\lambda_{i} x}, \quad x \geq 0$.

Hence the field variables can be written as the following:

$u=A_{1} x_{11} e^{-\lambda_{1} x}+A_{2} x_{21} e^{-\lambda_{2} x}+A_{3} x_{31} e^{-\lambda_{3} x}$,

$v=A_{1} x_{12} e^{-\lambda_{1} x}+A_{2} x_{22} e^{-\lambda_{2} x}+A_{3} x_{32} e^{-\lambda_{3} x}$,

$T=A_{1} x_{13} e^{-\lambda_{1} x}+A_{2} x_{23} e^{-\lambda_{2} x}+A_{3} x_{33} e^{-\lambda_{3} x}$.

The simplified form of Eqs. (21-23) can be written as:

$\sigma_{11}=A_{1} R_{11}(x)+A_{2} R_{12}(x)+A_{3} R_{13}(x)$,
$\sigma_{22}=A_{1} R_{21}(x)+A_{2} R_{22}(x)+A_{3} R_{23}(x)$,
$\sigma_{33}=A_{1} R_{31}(x)+A_{2} R_{32}(x)+A_{3} R_{33}(x)$,

where:

$R_{11}(x)=\left[-\lambda_{1} x_{11}+B_{1} \operatorname{iax}_{12}-x_{13}\right] e^{-\lambda_{1} x}$,

$R_{12}(x)=\left[-\lambda_{2} x_{21}+B_{1}\right.$ iax $\left._{22}-x_{23}\right] e^{-\lambda_{2} x}$,

$R_{13}(x)=\left[-\lambda_{3} x_{31}+B_{1} \operatorname{iax}_{32}-x_{33}\right] e^{-\lambda_{3} x}$,

$R_{21}(x)=\left[-\lambda_{1} B_{1} x_{11}+B_{2} \operatorname{iax}_{12}-B_{3} x_{13}\right] e^{-\lambda_{1} x}$,

$R_{21}(x)=\left[-\lambda_{2} B_{1} x_{21}+B_{2}\right.$ iax $\left._{22}-B_{3} x_{23}\right] e^{-\lambda_{2} x}$,

$R_{21}(x)=\left[-\lambda_{3} B_{1} x_{31}+B_{2} i a x_{32}-B_{3} x_{33}\right] e^{-\lambda_{3} x}$,

$R_{31}(x)=\left[B_{4}\right.$ iax $\left._{11}-\lambda_{1} x_{12}\right] e^{-\lambda_{1} x}$,

$R_{32}(x)=\left[B_{4}\right.$ iax $\left._{21}-\lambda_{2} x_{22}\right] e^{-\lambda_{2} x}$,

$R_{33}(x)=\left[B_{4}\right.$ iax $\left._{31}-\lambda_{3} x_{32}\right] e^{-\lambda_{3} x}$.

\section{Boundary conditions}

Considering the problem of a half-space $\phi$, defined as follows:

$\phi=(x, y, z): 0 \leq x \leq \infty, \quad-\infty \leq y \leq \infty, \quad-\infty \leq z \leq \infty$.

In order to determine the arbitrary constants $A_{i}^{\prime} s, i=1,2,3$, we consider the boundary conditions as follows.

\subsection{Case 1}

a) Mechanical Boundary condition:

For stress-free surface $x=0, \sigma_{11}=0, \sigma_{12}=0$.

b) Thermal Boundary condition:

$v T-\frac{d T}{d x}=r$

where $v$ is Biot's number. 


\subsection{Case 2}

a) Mechanical Boundary condition:

For stress-free surface $x=0, \sigma_{11}=-P_{1}+P_{2} e^{\omega t+i a y}, \sigma_{12}=0$.

b) Thermal Boundary condition:

$T=P_{3} e^{\omega t+i a y}$.

\section{Numerical analysis}

\subsection{Case 1}

\subsubsection{Distribution of different stress components}

Fig. 1 represents distribution of normal stress $\sigma_{11}$ for $y=0.3$.

For fixed time $t, \sigma_{11}$ gradually increases as $x$ increases. For fixed $x$ numerical values of $\sigma_{11}$ gradually decreases as $t$ increases.

Fig. 2 represents distribution of normal stress $\sigma_{12}$ for $y=0.2$.

For fixed time $t, \sigma_{12}$ gradually decreases as $x$ increases. For fixed x numerical values of $\sigma_{12}$ gradually increases as $t$ increases.

Fig. 3 represents distribution of normal stress $\sigma_{22}$ for $y=0.5$.

For fixed time $t, \sigma_{22}$ gradually decreases as $x$ increases. For fixed $x$ numerical values of $\sigma_{12}$ gradually increases as $t$ increases.

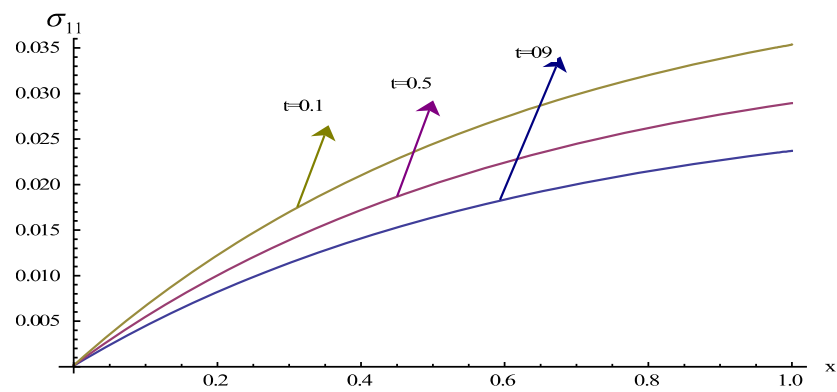

Fig. 1. Stress component $\sigma_{11}$ at $y=0.3$ for different values of $t$ verses $x$



Fig. 2. Stress component $\sigma_{12}$ at $y=0.2$ for different values of $t$ verses $x$

Fig. 4 represents distribution of normal stress $\sigma_{11}$ for different values of $x$ and $y$ for fixed $t=0.1$ and $\omega=0.5$.

$x$ numerical value of $\sigma_{11}$ gradually decreases as $y$ increases. For fixed $y$ the numerical value of $\sigma_{11}$ gradually increases as $x$ increases. $\sigma_{11}$ is maximum when $x=1$ and $y=0$.

Fig. 5 represent distribution of normal stress $\sigma_{12}$ for different values of $x$ and $y$ for fixed $t=0.4$ and $\omega=5$. 
For fixed $x$ numerical value of $\sigma_{12}$ gradually decreases as $y$ increases. For fixed $y$ the numerical value of $\sigma_{12}$ gradually decreases as $x$ increases. Significant changes occur in the region $0.2 \leq x \leq 0.6$ and $0.6 \leq y \leq 1.0$.

Fig. 6 represent distribution of stress component $\sigma_{22}$ at for different values of $x$ and $y$ for fixed $t=0.1$ and 0.1 .

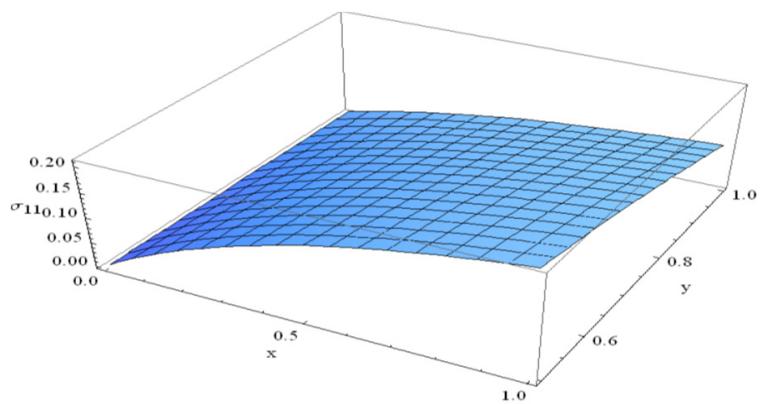

Fig. 4. Stress component $\sigma_{11}$ at $t=0.1$ and $\omega=0.5$ verses $x$ and $y$

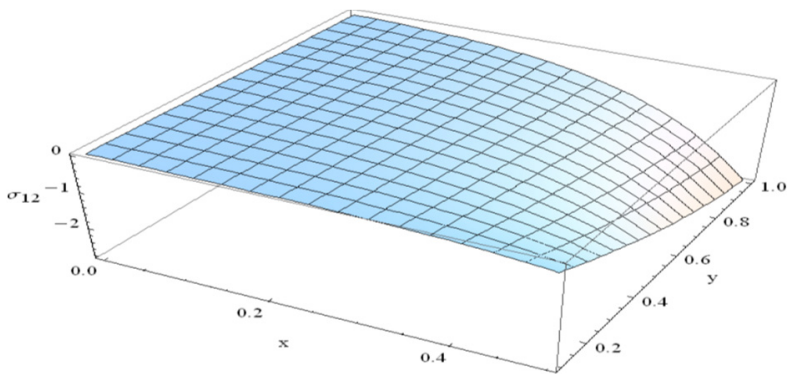

Fig. 5. The variation of stress component $\sigma_{12}$ at $t=0.4$ and $\omega=3$ verses $x$ and $y$

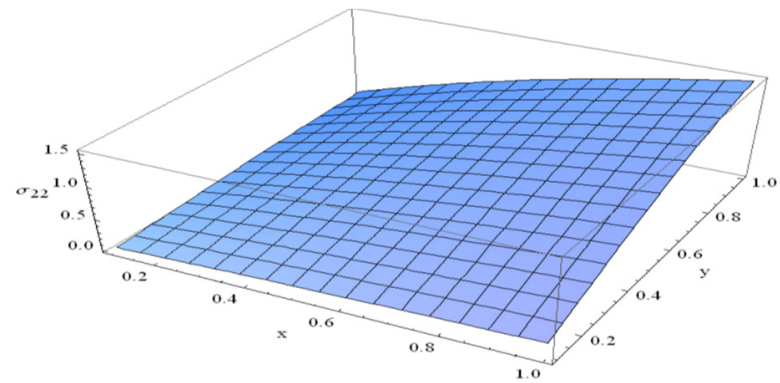

Fig. 6. Stress component $\sigma_{22}$ at $t=0.1$ and $\omega=0.1$ verses $x$ and $y$

For fixed $x$ numerical value of $\sigma_{22}$ gradually increases as $y$ increases. For fixed $y$ numerical value of $\sigma_{22}$ gradually increases as $x$ increases.

Fig. 7 represent distribution of normal stress $\sigma_{12}$ for different values of $x$ and $t$ for fixed $y=0.2$ and 1 .

For fixed $x$, nominal decreasing of numerical values of $\sigma_{12}$ has been seen as $t$ increases, while For fixed $t$, numerical values of $\sigma_{12}$ decreases gradually as $x$ increases. numerical values of $\sigma_{12}$ minimum at $x=1$ and $0.02 \leq t \leq 0.1$

Fig. 8 represent distribution of normal stress $\sigma_{22}$ for different values of $x$ and $t$ for fixed $y=0.5$ and 2 .

For fixed $x$, nominal decreasing of $\sigma_{22}$ has been seen as $\mathrm{t}$ increases. For fixed $t$, numerical values of $\sigma_{12}$ decreases as $x$ increases. Also, significant changes occur in the region $0.6 \leq x \leq 1.0$ and $0 \leq t \leq 1.0$. 


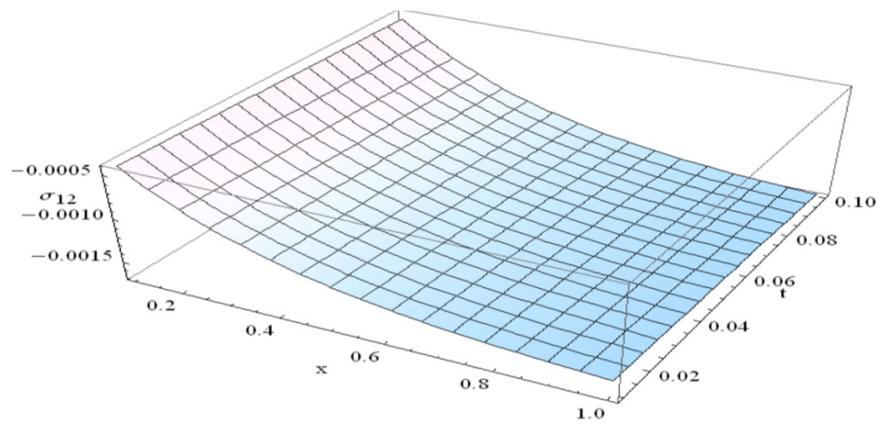

Fig. 7. Stress component $\sigma_{12}$ at $y=0.2$ and $\omega=1$ verses $x$ and $t$

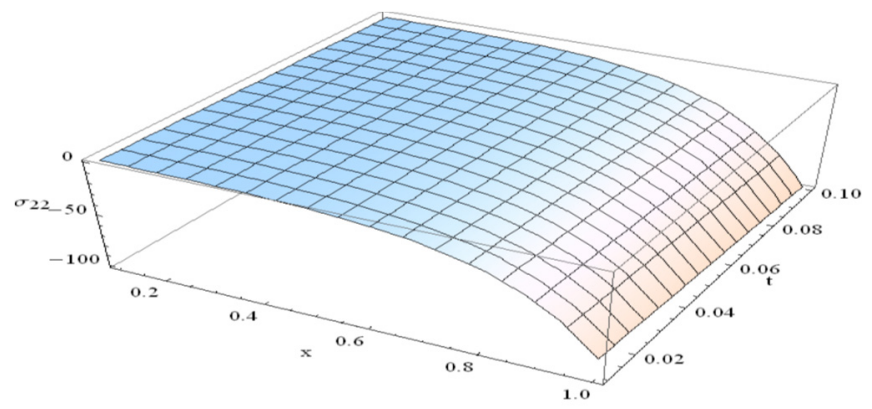

Fig. 8. The distribution of stress component $\sigma_{22}$ at $y=0.5$ and $\omega=2$ verses $x$ and $t$

\subsubsection{Distribution of temperature}

Fig. 9 represent distribution of temperature, $T$ for different values of $x$ and $y$ for fixed $t=0.3$ and 2.

For fixed $x$ numerical value of $T$ gradually decreases as $y$ increases. For fixed $y$ numerical value of $T$ gradually increases as $x$ increases. $T$ in minimum at $x=0$ and significant changes occurs in the region $0.6 \leq x \leq 1.0$ and $0 \leq y \leq 1.0$

Fig. 10 represent distribution of temperature, $T$ for different values of $x$ and $t$ for fixed $y=0.1$ and 1.5 .

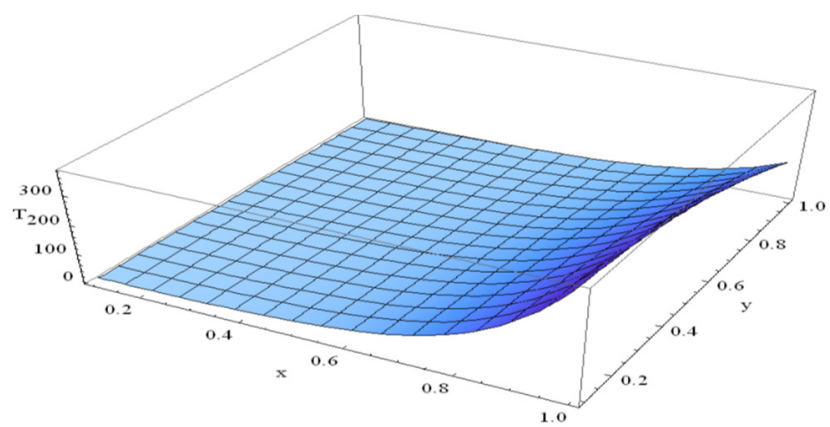

Fig. 9. The variation of $T$ at $t=0.3$ and $\omega=2$ verses $x$ and $y$

For fixed $t$ numerical value of $T$ nominally increases as $x$ increases. For fixed $x$ numerical value of $T$ gradually increases as $t$ increases.

Fig. 11 represent distribution of temperature, $T$ for different values of $y$ and $t$ for fixed $x=0.5$ and 3 .

For fixed $t$ numerical value of $T$ nominally increases as $y$ increases. For fixed $x$ numerical values of $T$ decreases as $t$ increases. 


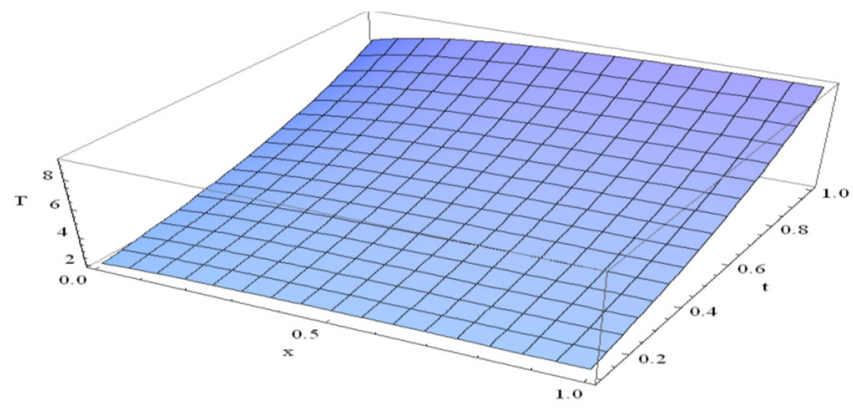

Fig. 10. Variation of $T$ at $y=0.1$ and $\omega=1.5$ verses $x$ and $t$

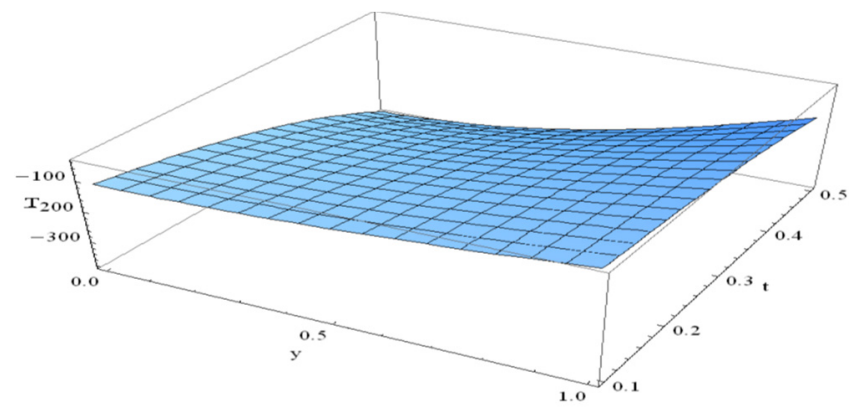

Fig. 11. Variation of $T$ at $x=0.5$ and $\omega=3$ verses $y$ and $t$

\subsection{Case 2}

\subsubsection{Distribution of different stress components}

Fig. 12 represents distribution of normal stress $\sigma_{11}$ for $y=0.3, t=0.01$ and 5 for different numerical values of $R_{p}$.

For fixed $R_{p}, \sigma_{11}$ gradually decreases as $x$ increases. For fixed x numerical values of $\sigma_{11}$ gradually decreases as $R_{p}$ increases.

Fig. 13 represents distribution of normal stress $\sigma_{11}$ for $y=0.3, t=0.01$ and 5 for different fractional values of $R_{p}$.

For fixed time $R_{p}, \sigma_{11}$ gradually increases as $x$ increases. For fixed $x$ numerical values of $\sigma_{11}$ gradually decreases as $R_{p}$ increases. Significant changes occurred for $0 \leq x \leq 0.4$.

Fig. 14 represents the distribution of stress component $\sigma_{12}$ at $y=0.2, t=0.03$ and 0.3 for different fractional values $R_{p}$ of verses $x$ for $P 1=1$.

For fixed time $R_{p}, \sigma_{11}$ gradually decreases as $x$ increases. For fixed x numerical values of $\sigma_{11}$ increases as $R_{p}$ increases. Significant changes occurred for $0 \leq x \leq 0.4$.

Fig. 15 represents the distribution of stress component $\sigma_{12}$ at $y=0.3, t=0.1$ and 4 for different integral values $R_{p}$ for $P 1=0$.

For fixed $R p, \sigma_{12}$ gradually decreases as $x$ increases. For fixed $x$ numerical values of $\sigma_{12}$ increases as $R_{p}$ increases.

Fig. 16 represents the distribution of stress component $\sigma_{22}$ at $y=0.2, t=0.6$ and 0.4 for different values $R_{p}$ for $P 1=0$.

For fixed $R_{p}=0.9, \sigma_{22}$ gradually increases as $x$ increases but for other fixed values of $R_{p}, \sigma_{22}$ gradually decreases as $x$ increases. For fixed $x, \sigma_{22}$ gradually increases as $x$ increases for $R_{p}=0.9$ but for other fixed values of $R_{p}, \sigma_{22}$ gradually decreases as $x$ increases.

Fig. 17 represents the distribution of stress component $\sigma_{11}$ for different values of $t$ for fixed $y=0.2$ and 1.5 . 


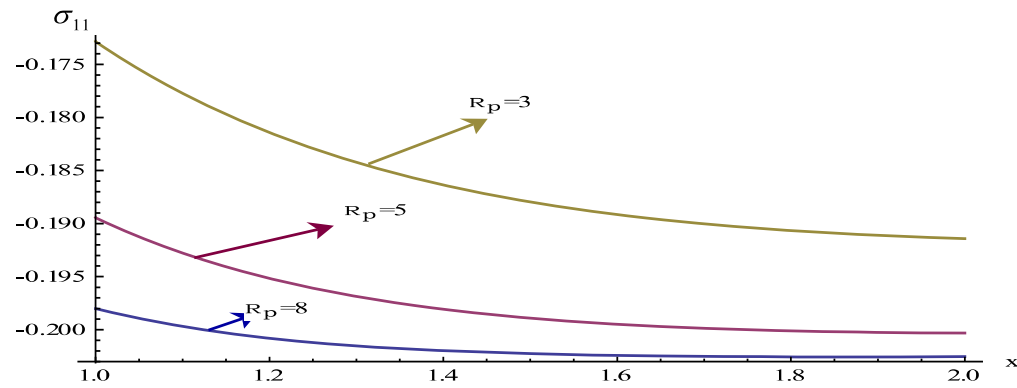

Fig. 12. Stress component $\sigma_{11}$ at $y=0.3, t=0.01$ and $\omega=5$ for different values $R_{p}$ of verses $x$

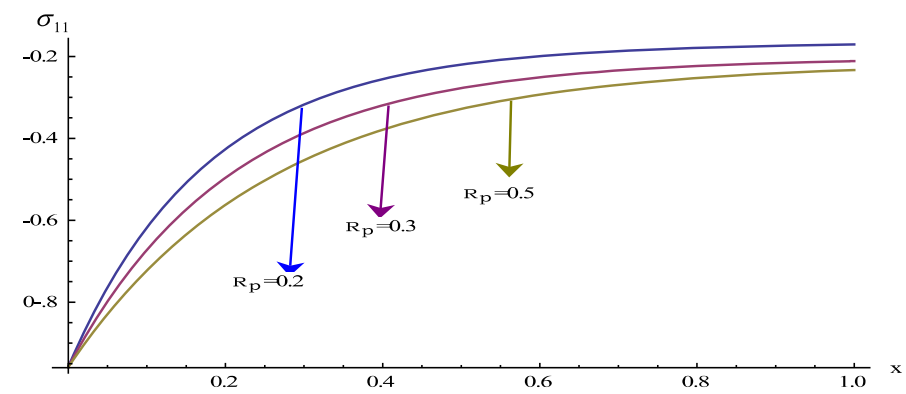

Fig. 13. Stress component $\sigma_{11}$ at $y=0.2$ and $\omega=0.2$ for different values $R_{p}$ of verses $x$

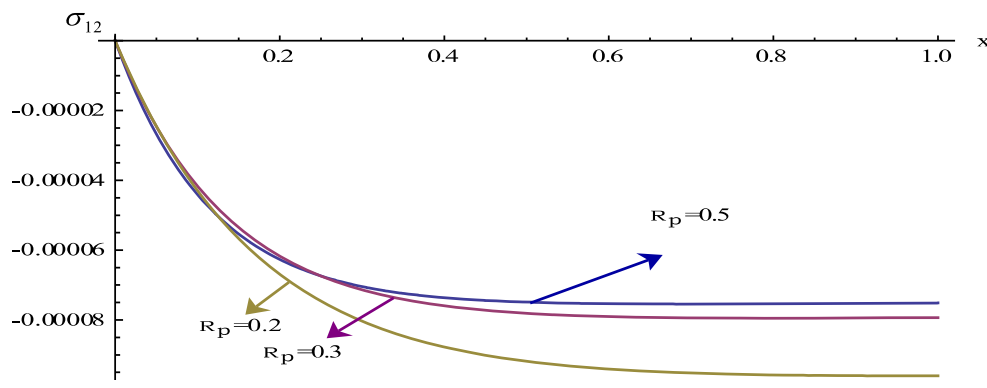

Fig. 14. Stress component $\sigma_{12}$ at $y=0.2, t=0.03$ and $\omega=0.3$ for different values $R_{p}$ of verses $x$ for $P 1=1$



Fig. 15. Stress component $\sigma_{12}$ at $y=0.3, t=0.1$ and $\omega=4$ for different values $R_{p}$ of verses $x$ for $P 1=0$

For fixed $t$, the numerical value of $\sigma_{11}$ gradually increases as $x$ increases. For fixed $x$, the numerical value of $\sigma_{11}$ gradually increases as $t$ increases.

Fig. 18 The distribution of stress component $\sigma_{12}$ for fixed $y=0.3$ and 0.5 for different values of $t$.

For fixed $t$, the numerical value of $\sigma_{12}$ gradually decreases as $x$ increases. For fixed $x$, the numerical value of $\sigma_{12}$ gradually increases as $t$ increases. 


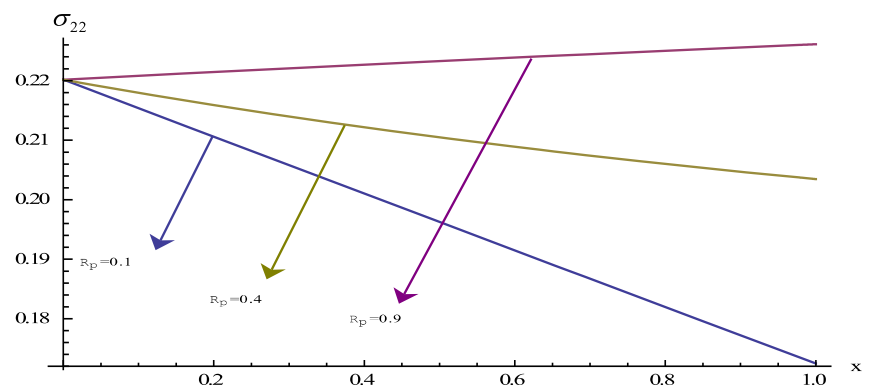

Fig. 16. Stress component $\sigma_{22}$ at $y=0.2, t=0.6$ and $\omega=0.4$ for different values $R_{p}$ of verses $x$ for $P 1=0$



Fig. 17. Stress component $\sigma_{11}$ at $y=0.2$ and $\omega=1.5$ for different values of $t$ verses $x$

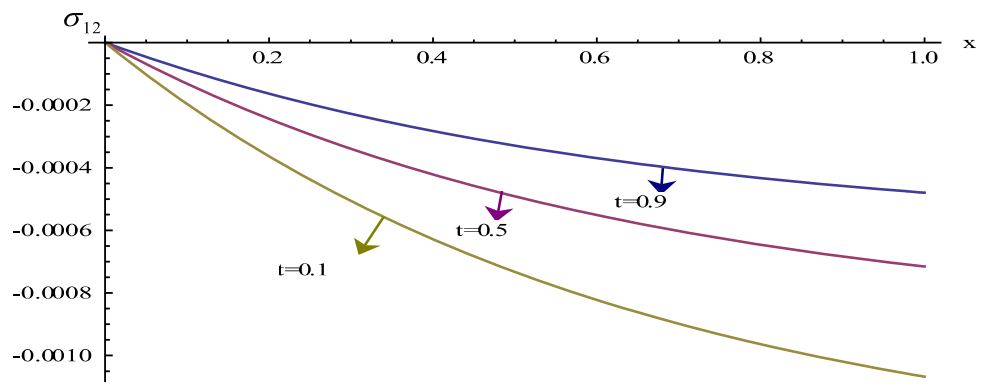

Fig. 18. Stress component $\sigma_{12}$ at $y=0.3$ and $\omega=0.5$ for different values of $t$ verses $x$

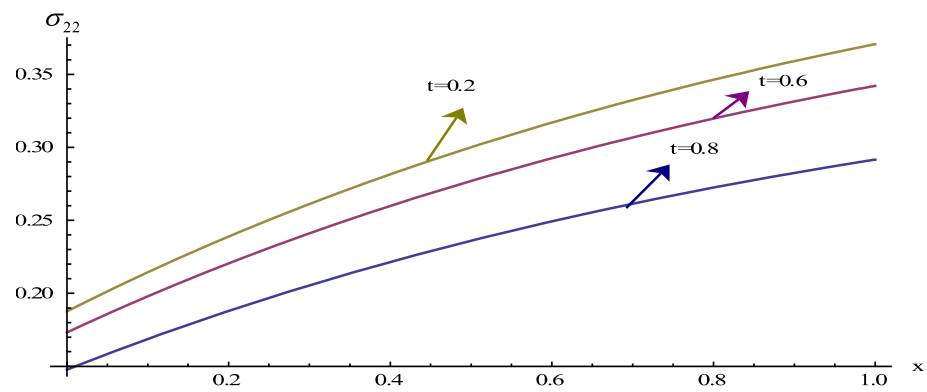

Fig. 19. Stress component $\sigma_{22}$ at $y=0.5$ and $\omega=0.2$ for different values of $t$ verses $x$

Fig. 19 represents the distribution of stress component $\sigma_{22}$ at fixed $y=0.5$ and 0.2 for different values of $t$.

For fixed $t$, the numerical value of $\sigma_{12}$ gradually increases as $x$ increases, but for fixed $x$, the numerical value of $\sigma_{12}$ gradually decreases as $t$ increases.

Fig. 20 represents distribution of normal stress $\sigma_{11}$ for different values of $x$ and for fixed 
$t=0.3$ and $y=0.3$.

For fixed $x$ numerical value of $\sigma_{11}$ remain constant as increases, but for fixed $\omega$ the numerical value of $\sigma_{11}$ gradually decreases as $x$ increases.

Fig. 21 represents distribution of normal stress $\sigma_{12}$ for different values of $x$ and for fixed $y=0.3$ and $t=0.5$.

For fixed $x$, nominal increasing of numerical values of $\sigma_{12}$ has been seen as increases, while for fixed $\omega$, numerical values of $\sigma_{12}$ increases gradually as $x$ increases after $x=0.6$ (approx.). Numerical values of $\sigma_{12}$ minimum at $x=0.5$ (approx.) and $1 \leq t \leq 3$.

Fig. 22 represents distribution of stress component $\sigma_{22}$ at for different values of $x$ and for fixed $t=0.1$ and $y=0.4$.

For fixed $\omega$ numerical value of $\sigma_{22}$ gradually decreases as $x$ increases. Numerical values of $\sigma_{22}$ minimum at $x=1$ and $0 \leq \omega \leq 1$.

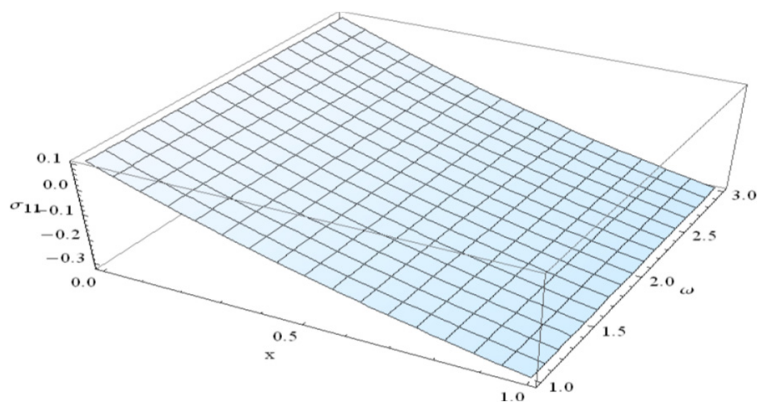

Fig. 20. Stress component $\sigma_{11}$ at $y=0.3$ and $t=0.3$ verses $x$ and $\omega$



Fig. 21. Stress component $\sigma_{12}$ at $y=0.3$ and $t=0.5$ verses $x$ and $\omega$

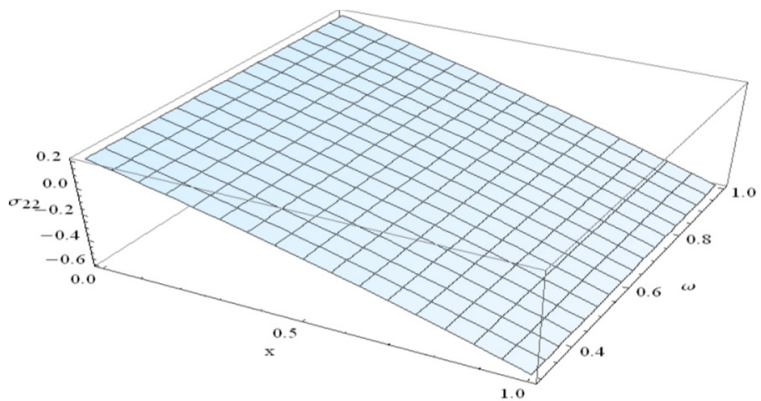

Fig. 22. Stress component $\sigma_{22}$ at $y=0.4$ and $t=0.1$ verses $x$ and $\omega$

\subsubsection{Distribution of temperature}

Fig. 23 represents distribution of temperature, $T$ for different values of $x$ and $\omega$ for fixed $t=0.1$ and $y=0.4$. 
For fixed $x$ numerical value of $T$ gradually increases in the region $0 \leq \omega \leq 0.3$ (approx.) For fixed $\omega$ numerical value of $T$ nominally increases as $x$ increases.

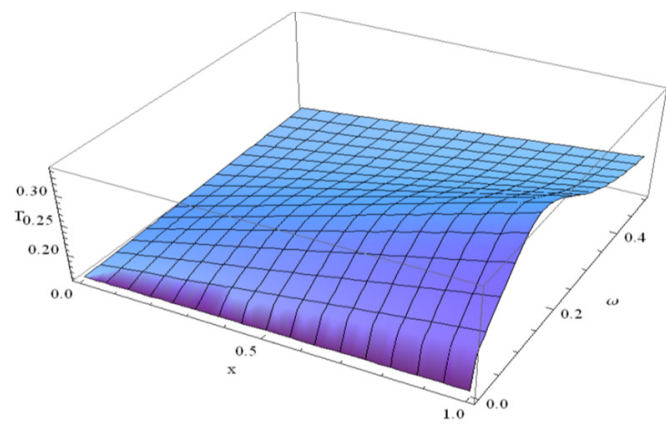

Fig. 23. The variation of $T$ at $y=0.4$ and $t=0.1$ verses $x$ and $\omega$

\section{Conclusion}

We consider the physical parameters in SI units given in Dhaliwal and Singh following below to obtain the numerical result to observe the effect of wave propagation:

$$
\begin{aligned}
& \rho=2660 \mathrm{~kg} / \mathrm{m}^{3}, \lambda=5.65 \times 10^{10} \mathrm{~N} / \mathrm{m}^{2}, \\
& \mu_{T}=2.46 \times 10^{10} \mathrm{~N} / \mathrm{m}^{2}, \quad \mu_{L}=5.66 \times 10^{10} \mathrm{~N} / \mathrm{m}^{2}, \\
& \alpha=-1.28 \times 10^{10} \mathrm{~N} / \mathrm{m}^{2}, \quad \beta=220.90 \times 10^{10} \mathrm{~N} / \mathrm{m}^{2}, \\
& \alpha_{11}=0.017 \times 10^{-4} \mathrm{deg}^{-1}, \alpha_{22}=0.015 \times 10^{-4} \mathrm{deg}^{-1}, \\
& l=0.5, \quad T_{0}=293 \mathrm{~K}, \quad c_{e}=0.787 \times 10^{3} \mathrm{Jg}^{-1} \mathrm{deg}^{-1} \\
& K_{11}=0.0921 \times 10^{10} \mathrm{Jm}^{-1} \mathrm{~s}^{-1} \mathrm{deg}^{-1} \\
& K_{22}=0.0963 \times 10^{10} \mathrm{Jm}^{-1} \mathrm{~s}^{-1} \mathrm{deg}^{-1} \\
& P_{1}=0 \text { or } 1, P_{2}=0.1, \quad P_{3}=0.2 .
\end{aligned}
$$

\section{References}

[1] Green W. A. Bending waves in strongly anisotropic plates. Quarterly Journal of Mechanics and Applied Mathematics, Vol. 35, 1982, p. 485-507.

[2] Abbas I. A., Othman M. I. A. Generalized thermoelastic interaction in a fiber-reinforced anisotropic half-space under hydrostatic initial stress. Journal of Vibration Control, Vol. 18, Issue 2, 2011, p. 175-182.

[3] Baylis E. R., Green W. A. Flexural waves in fiber reinforced laminated plates. Journal of Sound and Vibration, Vol. 110, 1986, p. 1-26.

[4] Rogerson G. A. Penetration of impact waves in a six-ply fiber composite laminate. Journal of Sound and Vibration, Vol. 158, 1992, p. 105-120.

[5] Dhaliwal R. S., Sherief H. H. Generalized thermoelasticity for anisotropic media. Quarterly of Applied Mathematics, Vol. 33, 1980, p. 1-8.

[6] Sherief H. H., El-Sayed A., El-Latief A. Fractional order theory of thermoelasticity. International Journal of Solids and Structures, Vol. 47, 2010, p. 269-275.

[7] Santra S., Das N. C., Kumar R., Lahir A. Three dimensional fractional order generalized thermoelastic problem under effect of rotation in a half space. Journal of Thermal Stresses, Vol. 38, 2015, p. 309-324.

[8] Bachher M., Sarkar N., Lahiri A. Generalized thermoelastic infinite medium with voids subjected to a instantaneous heat sources with fractional derivative heat transfer. International Journal of Mechanical Sciences, Vol. 89, 2014, p. 84-91.

[9] Lord H. W., Shulman Y. A generalized dynamical theory of thermoelasticity. Journal of the Mechanics and Physics of Solids, Vol. 15, 1967, p. 299-309.

[10] Green A. E., Lindsay K. A. Thermoelasticity. Journal of Elasticity, Vol. 2, 1972, p. 1-7. 
[11] Green A. E., Naghdi P. M. A re-examination of the basic results of thermomechanics. Proceedings of the Royal Society of London A, Vol. 432, 1991, p. 171-194.

[12] Green A. E., Naghdi P. M. On undamped heat waves in an elastic solid. Thermal Stresses, Vol. 15, 1992, p. 252-264.

[13] Green A. E., Naghdi P. M. Thermoelasticity without energy dissipation. Elasticity, Vol. 31, 1993, p. $189-208$

[14] Ibrahim A. Abbas Generalized magnetothermoelasticity in a fiber-reinforced anisotropic half-space. International Journal of Thermophysics, 2011, https://doi.org/10.1007/s10765-011-0957-3. 